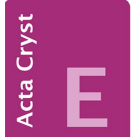
COMMUNICATIONS

ISSN 2056-9890

\section{Crystal structure of $(E)$-undec-2-enoic acid}

\section{Marcel Sonneck, Tim Peppel,* Anke Spannenberg and Sebastian Wohlrab}

Leibniz-Institut für Katalyse e. V. an der Universität Rostock, Albert-Einstein-Strasse 29a, 18059 Rostock, Germany. ${ }^{*}$ Correspondence e-mail: tim.peppel@catalysis.de

Received 13 May 2015; accepted 18 May 2015

Edited by C. Rizzoli, Universita degli Studi di Parma, Italy

In the molecule of the title low-melting $\alpha, \beta$-unsaturated carboxylic acid, $\mathrm{C}_{11} \mathrm{H}_{20} \mathrm{O}_{2}$, the least-squares mean line through the octyl chain forms an angle of $60.10(13)^{\circ}$ with the normal to plane of the acrylic acid fragment (r.m.s. deviation = $0.008 \AA$ ). In the crystal, centrosymmetrically related molecules are linked by pairs of $\mathrm{O}-\mathrm{H} \cdots \mathrm{O}$ hydrogen bonds into dimers, forming layers parallel to the (041) plane.

Keywords: crystal structure; hydrogen bond; dimer; unsaturated carboxylic acid.

CCDC reference: 1401589

\section{Related literature}

For an adapted direct synthesis of the title compound following the procedure established by Knoevenagel (1898) and Doebner (1902), see: Bikulova et al. (1988); Kemme et al. (2010). For crystal structure determinations of related unsaturated $\alpha, \beta$-carboxylic acids, see, for acrylic acid: Higgs \& Sass (1963); Chatani et al. (1963); Boese et al. (1999); Oswald \& Urquhart (2011); see, for crotonic acid: Shimizu et al. (1974); see, for (E)-pent-2-enoic acid: Peppel et al. (2015a); see, for (E)-hex-2-enoic acid: Peppel et al. (2015b). For structures of co-crystals containing $(E)$-hex-2-enoic acid, see: Aakeröy et al. (2003); Stanton \& Bak (2008).

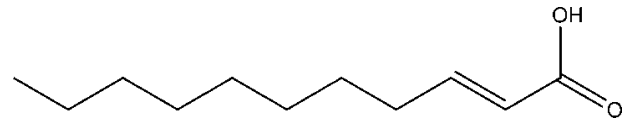

\section{Experimental}

2.1. Crystal data

$\mathrm{C}_{11} \mathrm{H}_{20} \mathrm{O}_{2} \quad M_{r}=184.27$
Triclinic, $P \overline{1}$

$a=4.6346(4) \AA$

$b=5.4200(5) \AA$

$c=22.7564$ (19) $\AA$

$\alpha=88.386$ (2)

$\beta=88.357$ (2)

$\gamma=78.340(2)^{\circ}$

\subsection{Data collection \\ Bruker APEXII CCD diffractometer (SADABS; Bruker, 2014) \\ $T_{\min }=0.87, T_{\max }=0.99$ \\ 2.3. Refinement \\ $R\left[F^{2}>2 \sigma\left(F^{2}\right)\right]=0.047$ \\ $w R\left(F^{2}\right)=0.134$ \\ $S=1.10$ \\ 2687 reflections \\ 122 parameters}

Absorption correction: multi-scan

$V=559.46(8) \AA^{3}$

$Z=2$

Mo $K \alpha$ radiation

$\mu=0.07 \mathrm{~mm}^{-1}$

$T=150 \mathrm{~K}$

$0.50 \times 0.41 \times 0.12 \mathrm{~mm}$

13660 measured reflections 2687 independent reflections 2317 reflections with $I>2 \sigma(I)$ $R_{\text {int }}=0.022$

Table 1

Hydrogen-bond geometry $\left(\AA,{ }^{\circ}\right)$.

\begin{tabular}{lllll}
\hline$D-\mathrm{H} \cdots A$ & $D-\mathrm{H}$ & $\mathrm{H} \cdots A$ & $D \cdots A$ & $D-\mathrm{H} \cdots A$ \\
\hline $\mathrm{O} 1-\mathrm{H} 1 \cdots \mathrm{O}^{\mathrm{i}}$ & $0.90(2)$ & $1.73(2)$ & $2.6244(14)$ & $172.2(19)$ \\
\hline
\end{tabular}

Symmetry code: (i) $-x+1,-y-1,-z+1$.

Data collection: APEX2 (Bruker, 2014); cell refinement: SAINT (Bruker, 2013); data reduction: SAINT; program(s) used to solve structure: SHELXS97 (Sheldrick, 2008); program(s) used to refine structure: SHELXL2014 (Sheldrick, 2015); molecular graphics: SHELXL2014; software used to prepare material for publication: SHELXL2014.

\section{Acknowledgements}

The authors thank P. Thiele (University of Rostock) for the DSC measurements and Professor Dr J. G. de Vries (LIKAT) for helpful support.

Supporting information for this paper is available from the IUCr electronic archives (Reference: RZ5160).

\section{References}

Aakeröy, C. B., Beatty, A. M., Helfrich, B. A. \& Nieuwenhuyzen, M. (2003). Cryst. Growth Des. 3, 159-165.

Bikulova, L. M., Verba, G. G., Kamaev, F. G. \& Abduvakhabov, A. A. (1988). Khim. Prir. Soedin. pp. 682-683.

Boese, R., Bläser, D., Steller, I., Latz, R. \& Bäumen, A. (1999). Acta Cryst. C55 IUC9900006.

Bruker (2013). SAINT. Bruker AXS Inc., Madison, Wisconsin, USA.

Bruker (2014). APEX2 and SADABS. Bruker AXS Inc., Madison, Wisconsin, USA.

Chatani, Y., Sakata, Y. \& Nitta, I. (1963). J. Polym. Sci. B Polym. Lett. 1, 419 421.

Doebner, O. (1902). Ber. Dtsch. Chem. Ges. 35, 1136-1147.

Higgs, M. A. \& Sass, R. L. (1963). Acta Cryst. 16, 657-661.

Kemme, S. T., Šmejkal, T. \& Breit, B. (2010). Chem. Eur. J. 16, 3423-3433.

Knoevenagel, E. (1898). Ber. Dtsch. Chem. Ges. 31, 2596-2619. 


\section{data reports}

Oswald, I. D. H. \& Urquhart, A. J. (2011). CrystEngComm, 13, 4503-4507.

Peppel, T., Sonneck, M., Spannenberg, A. \& Wohlrab, S. (2015a). Acta Cryst. E71, 0316.

Peppel, T., Sonneck, M., Spannenberg, A. \& Wohlrab, S. (2015b). Acta Cryst. E71, 0323 .
Sheldrick, G. M. (2008). Acta Cryst. A64, 112-122.

Sheldrick, G. M. (2015). Acta Cryst. C71, 3-8.

Shimizu, S., Kekka, S., Kashino, S. \& Haisa, M. (1974). Bull. Chem. Soc. Jpn, 47, 1627-1631.

Stanton, M. K. \& Bak, A. (2008). Cryst. Growth Des. 8, 3856-3862. 


\section{supporting information}

Acta Cryst. (2015). E71, o426-0427 [doi:10.1107/S2056989015009469]

\section{Crystal structure of $(E)$-undec-2-enoic acid}

\section{Marcel Sonneck, Tim Peppel, Anke Spannenberg and Sebastian Wohlrab}

\section{S1. Synthesis and crystallization}

Malonic acid (25.0g, $240.2 \mathrm{mmol}, 1.0 \mathrm{eq})$ is dissolved in dry pyridine $(38.0 \mathrm{~g}, 480.5 \mathrm{mmol}, 2.0 \mathrm{eq})$ at room temperature in a three-necked flask equipped with a magnetic stir bar and a reflux condenser under a mild flow of argon. Nonanal $(34.2 \mathrm{~g}, 240.2 \mathrm{mmol}, 1.0 \mathrm{eq})$ is then added in one portion and the resulting clear solution is further stirred for $72 \mathrm{~h}$ at room temperature under argon. Afterwards, the resulting light yellow to orange solution is brought to an acidic $\mathrm{pH}$ value by adding phosphoric acid at $0{ }^{\circ} \mathrm{C}(42.5 \mathrm{wt} . \%, 138.5 \mathrm{~g}, 600.6 \mathrm{mmol}, 2.5 \mathrm{eq})$. The resulting two layers are extracted three times with $150 \mathrm{~mL}$ portions of ethyl acetate and reduced to a volume of ca. $150 \mathrm{~mL}$ in vacuo. To remove impurities from aldol condensation the raw acid is converted into the corresponding sodium salt by addition of an aqueous solution of sodium carbonate $(20.4 \mathrm{~g}, 192.2 \mathrm{mmol}, 0.8 \mathrm{eq}$ in $200 \mathrm{~mL})$. After stirring for 30 minutes the water phase is separated and extracted three times with $150 \mathrm{~mL}$ portions of ethyl acetate. The water phase is then acidified with concentrated hydrochloric acid (37.0wt. \%, $35.5 \mathrm{~g}, 360.4 \mathrm{mmol}, 1.5 \mathrm{eq})$, the organic phase is separated and the water phase is again extracted three times with $150 \mathrm{~mL}$ portions of ethyl acetate. The combined organic phases are dried over $\mathrm{Na}_{2} \mathrm{SO}_{4}$ and evaporated to dryness under diminished pressure. The resulting raw product is further purified by distillation in vacuo yielding the product in purity $>99 \%(\mathrm{GC})$. m.p. $18^{\circ} \mathrm{C} .{ }^{1} \mathrm{H}$ NMR $\left(400 \mathrm{MHz}, \mathrm{CDCl}_{3}\right): \delta=12.24$ (br s, $\left.1 \mathrm{H}, \mathrm{OH}\right) ; 7.09\left(\mathrm{dt},{ }^{3} \mathrm{~J}\right.$ $\left.=15.6 \mathrm{~Hz},{ }^{3} J=7.0 \mathrm{~Hz}, 1 \mathrm{H},-\mathrm{CH}-\right) ; 5.82\left(\mathrm{dt},{ }^{3} J=15.6 \mathrm{~Hz},{ }^{4} J=1.6 \mathrm{~Hz}, 1 \mathrm{H},-\mathrm{CH}-\right) ; 2.26-2.19$ (m, 2H, $\left.-\mathrm{CH}_{2}-\right) ; 1.50-1.43$ (m, 2H, - $\left.\mathrm{CH}_{2}-\right)$; 1.33-1.24 (m, 10H, 5x - $\left.\mathrm{CH}_{2}-\right)$; 0.91-0.85 (m, 3H, - $\left.\mathrm{CH}_{3}-\right) .{ }^{13} \mathrm{C}^{\mathrm{NMR}}\left(100 \mathrm{MHz}, \mathrm{CDCl}_{3}\right): \delta=172.50(\mathrm{CO})$; $152.69(\mathrm{CH}) ; 120.76(\mathrm{CH}) ; 32.47\left(\mathrm{CH}_{2}\right) ; 31.98\left(\mathrm{CH}_{2}\right) ; 29.48\left(\mathrm{CH}_{2}\right), 29.32\left(\mathrm{CH}_{2}\right), 29.29\left(\mathrm{CH}_{2}\right) ; 28.02\left(\mathrm{CH}_{2}\right) ; 22.79\left(\mathrm{CH}_{2}\right)$; $14.22\left(\mathrm{CH}_{3}\right)$. MS (EI, 70eV): $m / z=184\left(\mathrm{M}^{+}, 0\right), 99$ (15), 97 (12), 96 (11), 95 (11), 86 (17), 84 (17), 83 (17), 82 (17), 81 (16), 73 (36), 70 (17), 69 (25), 68 (20), 67 (19), 57 (37), 56 (20), 55 (46), 54 (12), 53 (23), 45 (22), 43 (60), 42 (20), 41 (100), 40 (14), 39 (57), 29 (62). HRMS (ESI-TOF/MS): calculated for $\left.\mathrm{C}_{11} \mathrm{H}_{20} \mathrm{O}_{2}([\mathrm{M}-\mathrm{H}]]^{-}\right)$183.13905, found 183.13912. Elemental analysis for $\mathrm{C}_{11} \mathrm{H}_{20} \mathrm{O}_{2} \%$ (calc.): $\mathrm{C} 71.67$ (71.70); $\mathrm{H} 10.83$ (10.94). Suitable single crystals were grown by slow evaporation of an ethanolic solution at $-30^{\circ} \mathrm{C}$ over one week.

\section{S2. Refinement}

$\mathrm{H} 1$ could be found from the difference Fourier map and was refined with $U_{\text {iso }}(\mathrm{H})$ fixed at $1.5 U_{\text {eq }}(\mathrm{O})$. All other $\mathrm{H}$ atoms were placed in idealized positions with $\mathrm{d}(\mathrm{C}-\mathrm{H})=0.95 \AA(\mathrm{CH}), 0.99 \AA\left(\mathrm{CH}_{2}\right), 0.98 \AA\left(\mathrm{CH}_{3}\right)$ and refined using a riding model with $U_{\text {iso }}(\mathrm{H})$ fixed at $1.2 U_{\text {eq }}(\mathrm{C})$ for $\mathrm{CH}$ and $\mathrm{CH}_{2}$ and $1.5 U_{\text {eq }}(\mathrm{C})$ for $\mathrm{CH}_{3}$.

\section{S3. Comment}

The crystal structure of $(E)$-undec-2-enoic acid, $\mathrm{C}_{11} \mathrm{H}_{20} \mathrm{O}_{2}$, an $\alpha, \beta$-unsaturated carboxylic acid with a melting point near room temperature $\left(\mathrm{m} . \mathrm{p} .18^{\circ} \mathrm{C}\right)$, is characterized by acid dimers. The corresponding dimers are connected via intermolecular hydrogen bonds of the carboxylic groups $\mathrm{C}=\mathrm{O} \cdots \mathrm{H}-\mathrm{O}$. The crystal packing of $(E)$-undec-2-enoic acid is described by layers of acid dimers parallel to the $\left(\begin{array}{lll}0 & 4 & 1\end{array}\right)$ plane which are featured by layers of polar headgroups and 
hydrophobic hydrocarbon chains. The carboxylic group and the following three carbon atoms $(\mathrm{C} 2, \mathrm{C} 3, \mathrm{C} 4)$ of the $(E)-$ undec-2-enoic acid molecule lie in one plane (r.m.s. deviation $=0.008 \AA$ ), whereas the atoms of the hydrocarbon chain starting from $\mathrm{C} 4$ until $\mathrm{C} 11$ adopt a nearly fully staggered conformation.

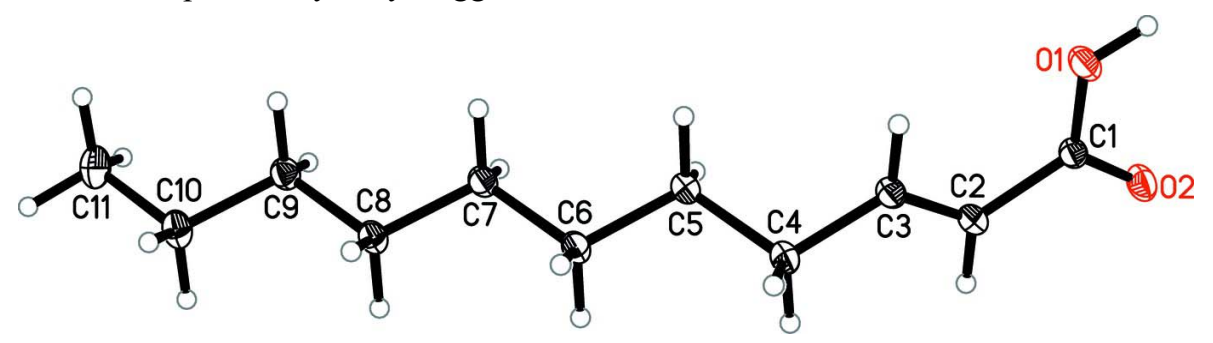

\section{Figure 1}

Molecular structure of the title compound with displacement ellipsoids drawn at 30\% probability level.

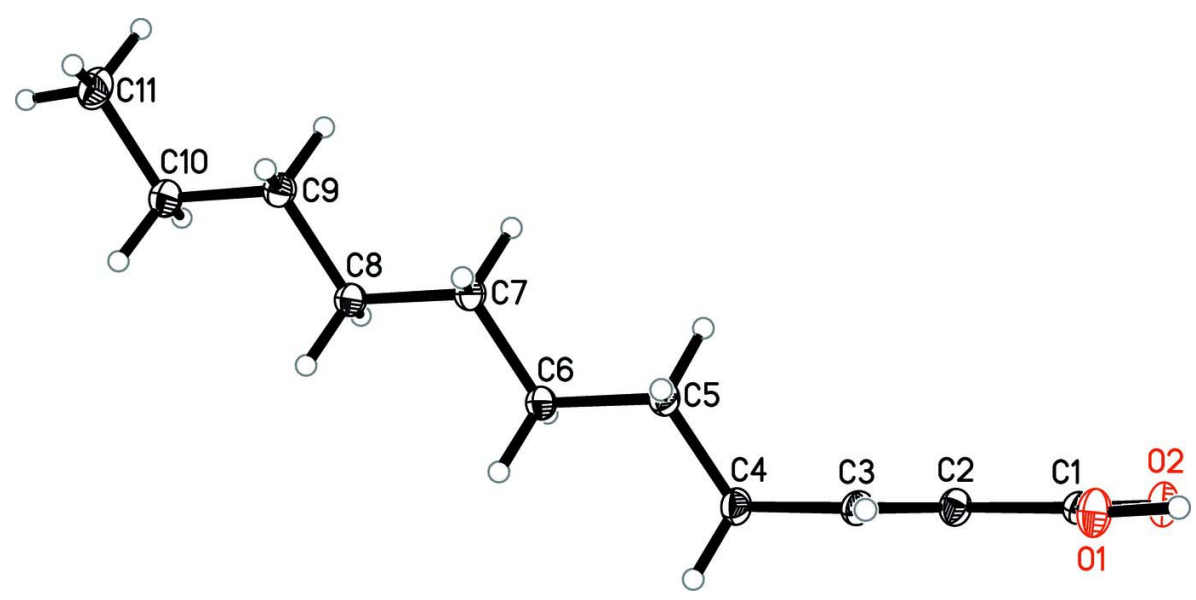

\section{Figure 2}

Side view of the molecular structure of the title compound (displacement ellipsoids drawn at $30 \%$ probability level).

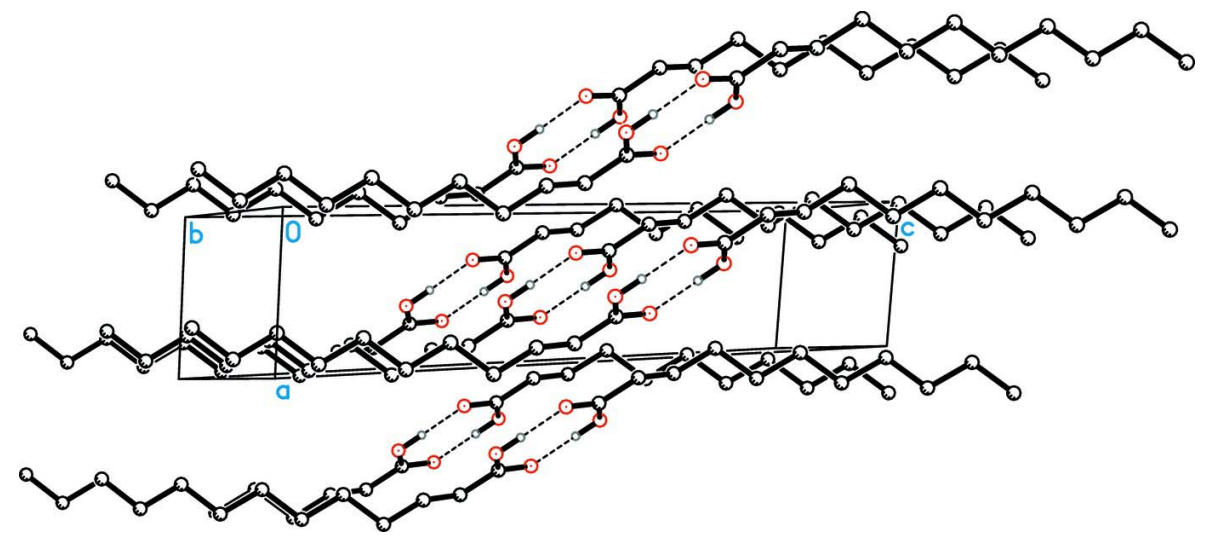

\section{Figure 3}

Packing diagram showing intermolecular $\mathrm{O}-\mathrm{H} \cdots \mathrm{O}$ hydrogen bonds. Hydrogen atoms not involved in hydrogen bonding are omitted for clarity. 


\section{(E)-Undec-2-enoic acid}

Crystal data

$$
\begin{aligned}
& \mathrm{C}_{11} \mathrm{H}_{20} \mathrm{O}_{2} \\
& M_{r}=184.27 \\
& \text { Triclinic, } P \overline{1} \\
& a=4.6346(4) \AA \\
& b=5.4200(5) \AA \\
& c=22.7564(19) \AA \\
& \alpha=88.386(2)^{\circ} \\
& \beta=88.357(2)^{\circ} \\
& \gamma=78.340(2)^{\circ} \\
& V=559.46(8) \AA^{3}
\end{aligned}
$$

\section{Data collection}

\section{Bruker APEXII CCD}

diffractometer

Radiation source: fine-focus sealed tube

Detector resolution: 8.3333 pixels $\mathrm{mm}^{-1}$

$\varphi$ and $\omega$ scans

Absorption correction: multi-scan

(SADABS; Bruker, 2014)

$T_{\min }=0.87, T_{\max }=0.99$

\section{Refinement}

Refinement on $F^{2}$

Least-squares matrix: full

$R\left[F^{2}>2 \sigma\left(F^{2}\right)\right]=0.047$

$w R\left(F^{2}\right)=0.134$

$S=1.10$

2687 reflections

122 parameters

0 restraints
$Z=2$

$F(000)=204$

$D_{\mathrm{x}}=1.094 \mathrm{Mg} \mathrm{m}^{-3}$

Mo $K \alpha$ radiation, $\lambda=0.71073 \AA$

Cell parameters from 7014 reflections

$\theta=2.7-29.0^{\circ}$

$\mu=0.07 \mathrm{~mm}^{-1}$

$T=150 \mathrm{~K}$

Plate, colourless

$0.50 \times 0.41 \times 0.12 \mathrm{~mm}$

13660 measured reflections

2687 independent reflections

2317 reflections with $I>2 \sigma(I)$

$R_{\text {int }}=0.022$

$\theta_{\text {max }}=28.0^{\circ}, \theta_{\min }=1.8^{\circ}$

$h=-6 \rightarrow 6$

$k=-7 \rightarrow 7$

$l=-30 \rightarrow 30$

Hydrogen site location: mixed

$\mathrm{H}$ atoms treated by a mixture of independent and constrained refinement

$w=1 /\left[\sigma^{2}\left(F_{\mathrm{o}}^{2}\right)+(0.0544 P)^{2}+0.245 P\right]$ where $P=\left(F_{\mathrm{o}}^{2}+2 F_{\mathrm{c}}^{2}\right) / 3$

$(\Delta / \sigma)_{\max }<0.001$

$\Delta \rho_{\max }=0.33$ e $\AA^{-3}$

$\Delta \rho_{\min }=-0.24 \mathrm{e} \AA^{-3}$

Special details

Geometry. All e.s.d.'s (except the e.s.d. in the dihedral angle between two 1.s. planes) are estimated using the full covariance matrix. The cell e.s.d.'s are taken into account individually in the estimation of e.s.d.'s in distances, angles and torsion angles; correlations between e.s.d.'s in cell parameters are only used when they are defined by crystal symmetry. An approximate (isotropic) treatment of cell e.s.d.'s is used for estimating e.s.d.'s involving 1.s. planes.

Fractional atomic coordinates and isotropic or equivalent isotropic displacement parameters $\left(\AA^{2}\right)$

\begin{tabular}{lllll}
\hline & $x$ & $y$ & $z$ & $U_{\text {iso }} * / U_{\text {eq }}$ \\
\hline $\mathrm{C} 1$ & $0.7148(3)$ & $-0.2848(2)$ & $0.45696(6)$ & $0.0231(3)$ \\
$\mathrm{C} 2$ & $0.8753(3)$ & $-0.1147(2)$ & $0.42422(6)$ & $0.0264(3)$ \\
$\mathrm{H} 2$ & 0.9723 & -0.0090 & 0.4457 & $0.032^{*}$ \\
$\mathrm{C} 3$ & $0.8904(3)$ & $-0.1026(2)$ & $0.36643(6)$ & $0.0251(3)$ \\
$\mathrm{H} 3$ & 0.7934 & -0.2107 & 0.3457 & $0.030^{*}$ \\
$\mathrm{C} 4$ & $1.0477(3)$ & $0.0674(3)$ & $0.33084(6)$ & $0.0278(3)$ \\
$\mathrm{H} 4 \mathrm{~A}$ & 1.2051 & -0.0358 & 0.3066 & $0.033^{*}$ \\
$\mathrm{H} 4 \mathrm{~B}$ & 1.1418 & 0.1667 & 0.3576 & $0.033^{*}$ \\
$\mathrm{C} 5$ & $0.8388(3)$ & $0.2471(2)$ & $0.29077(6)$ & $0.0247(3)$
\end{tabular}




\begin{tabular}{lllll} 
H5A & 0.7283 & 0.1481 & 0.2672 & $0.030^{*}$ \\
H5B & 0.6942 & 0.3622 & 0.3154 & $0.030^{*}$ \\
C6 & $0.9983(3)$ & $0.4033(2)$ & $0.24928(6)$ & $0.0248(3)$ \\
H6A & 1.1150 & 0.4972 & 0.2728 & $0.030^{*}$ \\
H6B & 1.1374 & 0.2884 & 0.2236 & $0.030^{*}$ \\
C7 & $0.7898(3)$ & $0.5893(2)$ & $0.21098(6)$ & $0.0249(3)$ \\
H7A & 0.6536 & 0.7060 & 0.2367 & $0.030^{*}$ \\
H7B & 0.6700 & 0.4953 & 0.1883 & $0.030^{*}$ \\
C8 & $0.9460(3)$ & $0.7429(2)$ & $0.16835(6)$ & $0.0263(3)$ \\
H8A & 1.0691 & 0.8340 & 0.1910 & $0.032^{*}$ \\
H8B & 1.0792 & 0.6263 & 0.1421 & $0.032^{*}$ \\
C9 & $0.7376(3)$ & $0.9326(2)$ & $0.13091(6)$ & $0.0267(3)$ \\
H9A & 0.6155 & 0.8415 & 0.1080 & $0.032^{*}$ \\
H9B & 0.6036 & 1.0487 & 0.1571 & $0.032^{*}$ \\
C10 & $0.8949(3)$ & $1.0861(3)$ & $0.08881(6)$ & $0.0329(3)$ \\
H10A & 1.0269 & 0.9703 & 0.0622 & $0.039^{*}$ \\
H10B & 1.0190 & 1.1756 & 0.1116 & $0.039^{*}$ \\
C11 & $0.6854(4)$ & $1.2777(3)$ & $0.05206(7)$ & $0.0392(4)$ \\
H11A & 0.5668 & 1.1901 & 0.0282 & $0.059^{*}$ \\
H11B & 0.7991 & 1.3723 & 0.0262 & $0.059^{*}$ \\
H11C & 0.5552 & 1.3943 & 0.0781 & $0.059^{*}$ \\
O1 & $0.5924(2)$ & $-0.43092(19)$ & $0.42681(4)$ & $0.0323(3)$ \\
O2 & $0.7045(2)$ & $-0.28190(19)$ & $0.51201(4)$ & $0.0320(3)$ \\
H1 & $0.490(5)$ & $-0.520(4)$ & $0.4504(9)$ & $0.048^{*}$ \\
& & & & \\
\hline
\end{tabular}

Atomic displacement parameters $\left(\AA^{2}\right)$

\begin{tabular}{lllllll}
\hline & $U^{11}$ & $U^{22}$ & $U^{33}$ & $U^{12}$ & $U^{13}$ & $U^{23}$ \\
\hline C1 & $0.0225(6)$ & $0.0211(6)$ & $0.0259(6)$ & $-0.0050(5)$ & $-0.0002(5)$ & $0.0019(5)$ \\
C2 & $0.0270(6)$ & $0.0256(6)$ & $0.0288(7)$ & $-0.0111(5)$ & $-0.0007(5)$ & $0.0023(5)$ \\
C3 & $0.0239(6)$ & $0.0227(6)$ & $0.0294(7)$ & $-0.0071(5)$ & $-0.0009(5)$ & $0.0031(5)$ \\
C4 & $0.0263(7)$ & $0.0299(7)$ & $0.0282(7)$ & $-0.0093(5)$ & $0.0012(5)$ & $0.0064(5)$ \\
C5 & $0.0245(6)$ & $0.0253(6)$ & $0.0255(6)$ & $-0.0084(5)$ & $0.0012(5)$ & $0.0022(5)$ \\
C6 & $0.0242(6)$ & $0.0239(6)$ & $0.0267(6)$ & $-0.0065(5)$ & $0.0029(5)$ & $0.0030(5)$ \\
C7 & $0.0243(6)$ & $0.0238(6)$ & $0.0269(6)$ & $-0.0065(5)$ & $0.0012(5)$ & $0.0023(5)$ \\
C8 & $0.0255(6)$ & $0.0239(6)$ & $0.0292(7)$ & $-0.0052(5)$ & $0.0028(5)$ & $0.0039(5)$ \\
C9 & $0.0271(7)$ & $0.0242(6)$ & $0.0288(7)$ & $-0.0058(5)$ & $0.0003(5)$ & $0.0030(5)$ \\
C10 & $0.0353(8)$ & $0.0307(7)$ & $0.0318(7)$ & $-0.0059(6)$ & $0.0037(6)$ & $0.0064(6)$ \\
C11 & $0.0485(9)$ & $0.0335(8)$ & $0.0338(8)$ & $-0.0053(7)$ & $-0.0004(7)$ & $0.0094(6)$ \\
O1 & $0.0394(6)$ & $0.0321(5)$ & $0.0302(5)$ & $-0.0195(4)$ & $-0.0007(4)$ & $0.0029(4)$ \\
O2 & $0.0413(6)$ & $0.0325(5)$ & $0.0253(5)$ & $-0.0157(4)$ & $0.0023(4)$ & $0.0032(4)$ \\
& & & & & & \\
\hline
\end{tabular}

Geometric parameters ( $\left.\AA,{ }^{\circ}\right)$

\begin{tabular}{llll}
\hline $\mathrm{C} 1-\mathrm{O} 2$ & $1.2527(16)$ & $\mathrm{C} 7-\mathrm{C} 8$ & $1.5219(17)$ \\
$\mathrm{C} 1-\mathrm{O} 1$ & $1.2862(16)$ & $\mathrm{C} 7-\mathrm{H} 7 \mathrm{~A}$ & 0.9900 \\
$\mathrm{C} 1-\mathrm{C} 2$ & $1.4717(17)$ & $\mathrm{C} 7-\mathrm{H} 7 \mathrm{~B}$ & 0.9900 \\
$\mathrm{C} 2-\mathrm{C} 3$ & $1.3153(19)$ & $\mathrm{C} 8-\mathrm{C} 9$ & $1.5207(18)$
\end{tabular}




\begin{tabular}{|c|c|c|c|}
\hline $\mathrm{C} 2-\mathrm{H} 2$ & 0.9500 & $\mathrm{C} 8-\mathrm{H} 8 \mathrm{~A}$ & 0.9900 \\
\hline $\mathrm{C} 3-\mathrm{C} 4$ & $1.4942(17)$ & $\mathrm{C} 8-\mathrm{H} 8 \mathrm{~B}$ & 0.9900 \\
\hline $\mathrm{C} 3-\mathrm{H} 3$ & 0.9500 & $\mathrm{C} 9-\mathrm{C} 10$ & $1.5173(19)$ \\
\hline $\mathrm{C} 4-\mathrm{C} 5$ & $1.5289(18)$ & C9-H9A & 0.9900 \\
\hline $\mathrm{C} 4-\mathrm{H} 4 \mathrm{~A}$ & 0.9900 & C9-H9B & 0.9900 \\
\hline $\mathrm{C} 4-\mathrm{H} 4 \mathrm{~B}$ & 0.9900 & $\mathrm{C} 10-\mathrm{C} 11$ & $1.520(2)$ \\
\hline $\mathrm{C} 5-\mathrm{C} 6$ & $1.5239(17)$ & $\mathrm{C} 10-\mathrm{H} 10 \mathrm{~A}$ & 0.9900 \\
\hline $\mathrm{C} 5-\mathrm{H} 5 \mathrm{~A}$ & 0.9900 & $\mathrm{C} 10-\mathrm{H} 10 \mathrm{~B}$ & 0.9900 \\
\hline C5-H5B & 0.9900 & $\mathrm{C} 11-\mathrm{H} 11 \mathrm{~A}$ & 0.9800 \\
\hline $\mathrm{C} 6-\mathrm{C} 7$ & $1.5215(17)$ & C11-H11B & 0.9800 \\
\hline C6-H6A & 0.9900 & $\mathrm{C} 11-\mathrm{H} 11 \mathrm{C}$ & 0.9800 \\
\hline C6-H6B & 0.9900 & $\mathrm{O} 1-\mathrm{H} 1$ & $0.90(2)$ \\
\hline $\mathrm{O} 2-\mathrm{C} 1-\mathrm{O} 1$ & $123.41(12)$ & $\mathrm{C} 8-\mathrm{C} 7-\mathrm{H} 7 \mathrm{~A}$ & 108.8 \\
\hline $\mathrm{O} 2-\mathrm{C} 1-\mathrm{C} 2$ & $119.25(11)$ & $\mathrm{C} 6-\mathrm{C} 7-\mathrm{H} 7 \mathrm{~B}$ & 108.8 \\
\hline $\mathrm{O} 1-\mathrm{C} 1-\mathrm{C} 2$ & $117.34(11)$ & $\mathrm{C} 8-\mathrm{C} 7-\mathrm{H} 7 \mathrm{~B}$ & 108.8 \\
\hline $\mathrm{C} 3-\mathrm{C} 2-\mathrm{C} 1$ & $122.85(12)$ & $\mathrm{H} 7 \mathrm{~A}-\mathrm{C} 7-\mathrm{H} 7 \mathrm{~B}$ & 107.7 \\
\hline $\mathrm{C} 3-\mathrm{C} 2-\mathrm{H} 2$ & 118.6 & $\mathrm{C} 9-\mathrm{C} 8-\mathrm{C} 7$ & $113.76(11)$ \\
\hline $\mathrm{C} 1-\mathrm{C} 2-\mathrm{H} 2$ & 118.6 & $\mathrm{C} 9-\mathrm{C} 8-\mathrm{H} 8 \mathrm{~A}$ & 108.8 \\
\hline $\mathrm{C} 2-\mathrm{C} 3-\mathrm{C} 4$ & $125.24(12)$ & $\mathrm{C} 7-\mathrm{C} 8-\mathrm{H} 8 \mathrm{~A}$ & 108.8 \\
\hline $\mathrm{C} 2-\mathrm{C} 3-\mathrm{H} 3$ & 117.4 & $\mathrm{C} 9-\mathrm{C} 8-\mathrm{H} 8 \mathrm{~B}$ & 108.8 \\
\hline $\mathrm{C} 4-\mathrm{C} 3-\mathrm{H} 3$ & 117.4 & $\mathrm{C} 7-\mathrm{C} 8-\mathrm{H} 8 \mathrm{~B}$ & 108.8 \\
\hline $\mathrm{C} 3-\mathrm{C} 4-\mathrm{C} 5$ & $111.88(11)$ & $\mathrm{H} 8 \mathrm{~A}-\mathrm{C} 8-\mathrm{H} 8 \mathrm{~B}$ & 107.7 \\
\hline $\mathrm{C} 3-\mathrm{C} 4-\mathrm{H} 4 \mathrm{~A}$ & 109.2 & $\mathrm{C} 10-\mathrm{C} 9-\mathrm{C} 8$ & $113.44(11)$ \\
\hline $\mathrm{C} 5-\mathrm{C} 4-\mathrm{H} 4 \mathrm{~A}$ & 109.2 & $\mathrm{C} 10-\mathrm{C} 9-\mathrm{H} 9 \mathrm{~A}$ & 108.9 \\
\hline $\mathrm{C} 3-\mathrm{C} 4-\mathrm{H} 4 \mathrm{~B}$ & 109.2 & $\mathrm{C} 8-\mathrm{C} 9-\mathrm{H} 9 \mathrm{~A}$ & 108.9 \\
\hline $\mathrm{C} 5-\mathrm{C} 4-\mathrm{H} 4 \mathrm{~B}$ & 109.2 & $\mathrm{C} 10-\mathrm{C} 9-\mathrm{H} 9 \mathrm{~B}$ & 108.9 \\
\hline $\mathrm{H} 4 \mathrm{~A}-\mathrm{C} 4-\mathrm{H} 4 \mathrm{~B}$ & 107.9 & $\mathrm{C} 8-\mathrm{C} 9-\mathrm{H} 9 \mathrm{~B}$ & 108.9 \\
\hline $\mathrm{C} 6-\mathrm{C} 5-\mathrm{C} 4$ & $112.95(11)$ & $\mathrm{H} 9 \mathrm{~A}-\mathrm{C} 9-\mathrm{H} 9 \mathrm{~B}$ & 107.7 \\
\hline $\mathrm{C} 6-\mathrm{C} 5-\mathrm{H} 5 \mathrm{~A}$ & 109.0 & $\mathrm{C} 9-\mathrm{C} 10-\mathrm{C} 11$ & $113.21(13)$ \\
\hline $\mathrm{C} 4-\mathrm{C} 5-\mathrm{H} 5 \mathrm{~A}$ & 109.0 & $\mathrm{C} 9-\mathrm{C} 10-\mathrm{H} 10 \mathrm{~A}$ & 108.9 \\
\hline $\mathrm{C} 6-\mathrm{C} 5-\mathrm{H} 5 \mathrm{~B}$ & 109.0 & $\mathrm{C} 11-\mathrm{C} 10-\mathrm{H} 10 \mathrm{~A}$ & 108.9 \\
\hline $\mathrm{C} 4-\mathrm{C} 5-\mathrm{H} 5 \mathrm{~B}$ & 109.0 & $\mathrm{C} 9-\mathrm{C} 10-\mathrm{H} 10 \mathrm{~B}$ & 108.9 \\
\hline $\mathrm{H} 5 \mathrm{~A}-\mathrm{C} 5-\mathrm{H} 5 \mathrm{~B}$ & 107.8 & $\mathrm{C} 11-\mathrm{C} 10-\mathrm{H} 10 \mathrm{~B}$ & 108.9 \\
\hline $\mathrm{C} 7-\mathrm{C} 6-\mathrm{C} 5$ & $113.02(11)$ & $\mathrm{H} 10 \mathrm{~A}-\mathrm{C} 10-\mathrm{H} 10 \mathrm{~B}$ & 107.8 \\
\hline $\mathrm{C} 7-\mathrm{C} 6-\mathrm{H} 6 \mathrm{~A}$ & 109.0 & $\mathrm{C} 10-\mathrm{C} 11-\mathrm{H} 11 \mathrm{~A}$ & 109.5 \\
\hline $\mathrm{C} 5-\mathrm{C} 6-\mathrm{H} 6 \mathrm{~A}$ & 109.0 & $\mathrm{C} 10-\mathrm{C} 11-\mathrm{H} 11 \mathrm{~B}$ & 109.5 \\
\hline $\mathrm{C} 7-\mathrm{C} 6-\mathrm{H} 6 \mathrm{~B}$ & 109.0 & $\mathrm{H} 11 \mathrm{~A}-\mathrm{C} 11-\mathrm{H} 11 \mathrm{~B}$ & 109.5 \\
\hline $\mathrm{C} 5-\mathrm{C} 6-\mathrm{H} 6 \mathrm{~B}$ & 109.0 & $\mathrm{C} 10-\mathrm{C} 11-\mathrm{H} 11 \mathrm{C}$ & 109.5 \\
\hline $\mathrm{H} 6 \mathrm{~A}-\mathrm{C} 6-\mathrm{H} 6 \mathrm{~B}$ & 107.8 & $\mathrm{H} 11 \mathrm{~A}-\mathrm{C} 11-\mathrm{H} 11 \mathrm{C}$ & 109.5 \\
\hline $\mathrm{C} 6-\mathrm{C} 7-\mathrm{C} 8$ & $113.71(11)$ & $\mathrm{H} 11 \mathrm{~B}-\mathrm{C} 11-\mathrm{H} 11 \mathrm{C}$ & 109.5 \\
\hline $\mathrm{C} 6-\mathrm{C} 7-\mathrm{H} 7 \mathrm{~A}$ & 108.8 & $\mathrm{C} 1-\mathrm{O} 1-\mathrm{H} 1$ & $110.8(13)$ \\
\hline $\mathrm{O} 2-\mathrm{C} 1-\mathrm{C} 2-\mathrm{C} 3$ & $-178.40(13)$ & $\mathrm{C} 4-\mathrm{C} 5-\mathrm{C} 6-\mathrm{C} 7$ & $-177.83(11)$ \\
\hline $\mathrm{O} 1-\mathrm{C} 1-\mathrm{C} 2-\mathrm{C} 3$ & 1.88 (19) & $\mathrm{C} 5-\mathrm{C} 6-\mathrm{C} 7-\mathrm{C} 8$ & $-178.77(11)$ \\
\hline $\mathrm{C} 1-\mathrm{C} 2-\mathrm{C} 3-\mathrm{C} 4$ & $179.50(12)$ & $\mathrm{C} 6-\mathrm{C} 7-\mathrm{C} 8-\mathrm{C} 9$ & $-178.82(11)$ \\
\hline $\mathrm{C} 2-\mathrm{C} 3-\mathrm{C} 4-\mathrm{C} 5$ & $-119.96(15)$ & $\mathrm{C} 7-\mathrm{C} 8-\mathrm{C} 9-\mathrm{C} 10$ & $179.63(11)$ \\
\hline $\mathrm{C} 3-\mathrm{C} 4-\mathrm{C} 5-\mathrm{C} 6$ & $-173.87(11)$ & $\mathrm{C} 8-\mathrm{C} 9-\mathrm{C} 10-\mathrm{C} 11$ & $-179.27(12)$ \\
\hline
\end{tabular}


supporting information

Hydrogen-bond geometry $\left(\AA,{ }^{\circ}\right)$

\begin{tabular}{lllll}
\hline$D-\mathrm{H} \cdots A$ & $D-\mathrm{H}$ & $\mathrm{H} \cdots A$ & $D \cdots A$ & $D-\mathrm{H} \cdots A$ \\
\hline $\mathrm{O} 1-\mathrm{H} 1 \cdots \mathrm{O} 2^{\mathrm{i}}$ & $0.90(2)$ & $1.73(2)$ & $2.6244(14)$ & $172.2(19)$ \\
\hline
\end{tabular}

Symmetry code: (i) $-x+1,-y-1,-z+1$. 